\title{
Controlling the Charge and Organization of Anionic Lipid Bilayers: Effect of Monovalent and Divalent Ions
}

\author{
Emily R. Lamberson, Lee R. Cambrea, and Jennifer S. Hovis*
}

Department of Chemistry, Purdue University, West Lafayette, IN 47907-2018

\section{Supporting Information}

\section{Experimental Methods}

Materials: Chloroform stock solutions of 1,2-dioleoyl-sn-glycero-3-phosphocholine (DOPC), 1,2-dioleoyl-sn-glycero-3-phosphate (DOPA), and 1-palmitoyl-2-[6-[(7-nitro-21,3-benzoxadiazol-4-yl)amino]hexanoyl]-sn-glycero-3-phosphocholine (NBD PC) were purchased from Avanti Polar Lipids, Inc. and used without further purification. $N$-(Texas Red sulfonyl)-1,2-dihexadecanoyl-sn-glycero-3-phosphoethanolamine (TR-DHPE) and CoverWell $^{\mathrm{TM}}$ perfusion chamber gaskets were purchased from Invitrogen-Molecular Probes, Inc. Glass coverslips, $22 \times 30$ \#1.5 were purchased from Fisher Scientific, and ICN 7X detergent was purchased from MP Biomedicals, Inc. 4-(2-hydroxyethyl)-1piperazineethanesulfonic acid (HEPES) and 2-(N-morpholino)ethanesulfonic acid hydrate (MES hydrate) were purchased from Sigma Chemical Co. Potassium chloride $(\mathrm{KCl})$, potassium iodide $(\mathrm{KI})$, potassium hydroxide $(\mathrm{KOH})$, and calcium chloride dihydrate $\left(\mathrm{CaCl}_{2}\right)$ were purchased from Mallinckrodt Chemicals.

Vesicle and supported lipid bilayer preparation: Large unilamellar vesicles (LUVs) were prepared by the extrusion method. Briefly, the chloroform solvated lipids were mixed at the appropriate molar ratios, dried under nitrogen, and held under vacuum for 1 hour; the dried lipids were rehydrated in a $50 \mathrm{mM}$ MES hydrate, $250 \mathrm{mM} \mathrm{KCl} \mathrm{buffer,} \mathrm{pH} 5(\mathrm{pH}$ adjusted with concentrated $\mathrm{KOH}$ ). The lipid suspension was then extruded 21 times through a polycarbonate membrane with $50 \mathrm{~nm}$ pores. Following extrusion, the LUV solution was centrifuged for 5 minutes at 14,000 rpm (Eppendorf Minispin Plus). The extruded vesicles were stored at $20^{\circ} \mathrm{C}$, shielded from light, and used within $12 \mathrm{hr}$. Supported lipid bilayers were formed, by vesicle fusion, inside a $60 \mu \mathrm{L}$ perfusion chamber on glass slides that had been washed in dilute ICN $7 \mathrm{X}$ detergent, rinsed extensively in $18 \mathrm{M} \Omega$-cm water, and baked at $450{ }^{\circ} \mathrm{C}$ for 4 hours (slides were used within a day of preparation). After 5 minutes, excess vesicles were removed by flushing the perfusion chamber with the same buffer used in vesicle preparation. The bulk solution was then exchanged with a buffer composed of 50mM HEPES and $250 \mathrm{mM} \mathrm{KCl}$, adjusted to $\mathrm{pH} 7.0$ or 8.0 with concentrated $\mathrm{KOH}$. Fluid-fluid separation was initiated using an exchange buffer composed of $50 \mathrm{mM}$ HEPES, $0-250 \mathrm{mM} \mathrm{KCl}$ and $0-5 \mathrm{mM}$ $\mathrm{CaCl}_{2}, \mathrm{pH} 7.0$ or 8.0 .

Imaging of the supported lipid bilayer: A Nikon TE2000 fluorescence microscope equipped with a Cascade 512B or Cascade 650 CCD camera (Roper Scientific) was used to image the bilayers. An X-Cite 120 arc lamp (EXFO) was used as a light source. The 
NBD and Texas Red DHPE fluorophores were imaged using appropriate filter cube sets (Chroma Technology Corp.). Images were acquired using a 40X, 1.30 NA objective.

Image Quantification: Raw images were cropped to $80 \mu \mathrm{m} \times 80 \mu \mathrm{m}$ and thresholded using a MatLab script. The threshold limit was set such that the PA-rich regions were below the limit and the PC-rich regions were above the limit. For bilayers that has white dots, a second threshold limit was set above the PC-rich region intensity and below the white dot intensity. The intensities above this higher threshold limit were discarded. To calculate the percent area coverage of PA-rich regions, the number of pixels whose intensity was below the lower threshold limit were summed and divided by the total number of pixels in the image. To calculate the percent fluorophore depletion, the average intensities of all the pixels below the lowest threshold limit (PA-rich regions) and between the lower and higher threshold limits (PC-rich regions) respectively were calculated. From all the images, appropriate backgrounds (dark noise of the camera, glass autofluorescence, etc) were subtracted off using counts obtained from a perfusion chamber containing the appropriate buffer. For some of the $\mathrm{K}^{+} / \mathrm{Ca}^{2+}$ ratios, the PA-rich regions were widely spaced. To avoid overestimating the percent area, we randomly sampled 6-10 spots on 3-5 samples.

Quenching experiments: Bilayers were formed and phase separation induced as described above. An image was recorded, the bulk solution was exchanged for one containing 500 $\mathrm{mM} \mathrm{KI}$ instead of $\mathrm{KCl}$, and a second image was recorded. The perfusion chamber was then rinsed with the original buffer, and a final image was recorded.

Fluorescence recovery after photobleaching (FRAP): The FRAP measurement system has been described in detail elsewhere. (cite) Briefly, a Nikon TE2000 fluorescence microscope equipped with a 40x oil immersion objective, a NBD filter set (Chroma Technology Corp.) and a silicon avalanche photodiode (APD) Single Photon Counting Module (SPCM-AQR-16-FC,PerkinElmer, Inc., Vaudreuil, Quebec) was used to focus, collect and count the emitted fluorescence. A $15 \mathrm{~mW}$ Argon ion laser (488 nm Melles Griot), which was attenuated to $150 \mathrm{nW}$ using a $5 \mathrm{X}$ (focal transmission of $1 \times 10^{5}$ ) neutral density filter (NE50B, Thorlabs, Inc., Newton, NJ), was used to monitor the initial intensity of the bilayer. The neutral density filter was removed from the beam path for one second and the bilayer was bleached to background. The bleach spot was $\sim 12 \mu \mathrm{m}$ in diameter. The neutral density filter was then replaced and the counts monitored until the intensity recovered to $>95 \%$ of the original intensity.

\section{Supplemental Figures}

Fluorescence Recovery after Photobleaching (FRAP): The mobility of phase separated bilayers was confirmed by FRAP. An image of the bilayer was recorded immediately before (S1b), immediately after (S1c), and 15 minutes after (S1d) bleaching. The bilayer was then bleached to background and the recovery monitored as described above (S1a). The intensity data was normalized to data acquired prior to bleaching. The intensity recovery is $>95 \%$, indicating that the lipids are diffusing on time-scales consistent with a fluid bilayer. 


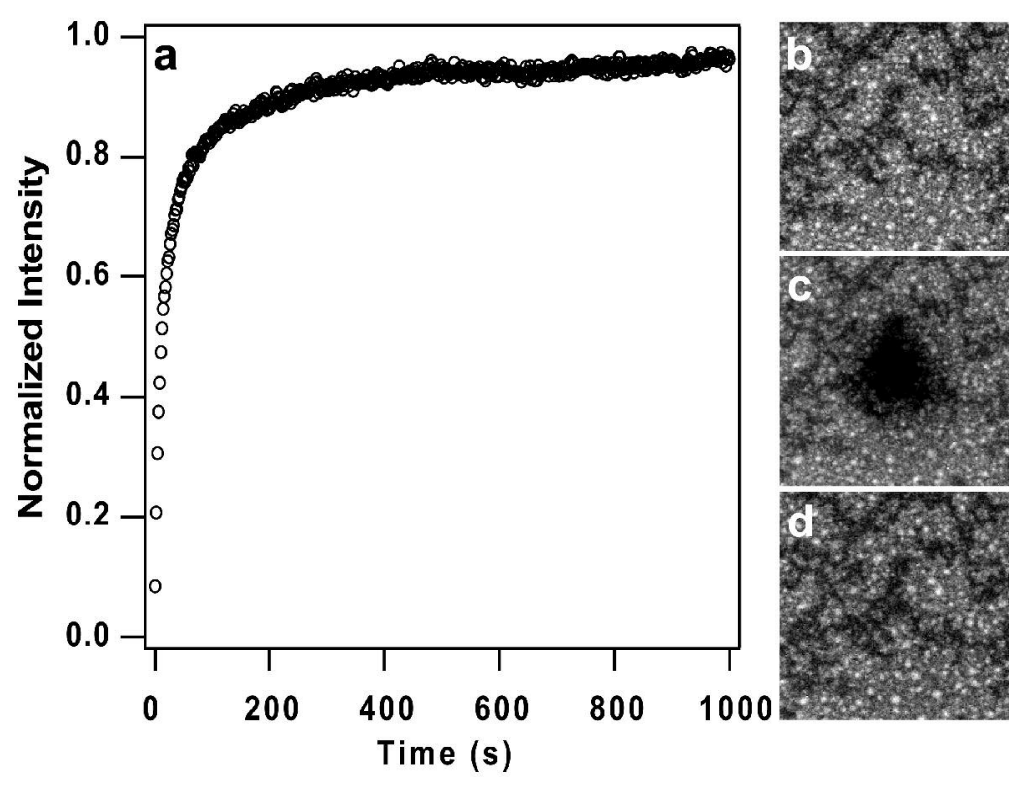

Figure S1: Bilayers composed of $30 \mathrm{~mol} \%$ DOPA, $69 \mathrm{~mol} \%$ DOPC, and $1 \mathrm{~mol} \% \mathrm{NBD}$ $\mathrm{PC}$ were formed and the bulk solution was exchanged with a $0 \mathrm{mM} \mathrm{KCl}, 0.5 \mathrm{mM} \mathrm{CaCl}_{2}$ buffer, $\mathrm{pH}$ 8. (a) Plot of fluorescence recovery as a function of time. (b) Epi-fluorescence image of the bilayer prior to bleaching, (c) immediately after bleaching, and (d) $\sim 15$ minutes after bleaching. Images and intensities were collected using a 40x, 1.3 NA objective, and images (b-d) are $60 \mu \mathrm{m} \times 60 \mu \mathrm{m}$.

Quenching Experiments: To investigate whether both bilayer leaflets phase-separated in response to changing $\mathrm{K}^{+}$and $\mathrm{Ca}^{2+}$ concentrations, iodide was used to quench the top leaflet of a head-labeled Texas Red bilayer, as described above. Figure S2 shows the bilayer (a) before phase separation, (b) after phase separation induced by a $50 \mathrm{mM} \mathrm{KCl}$, $0.5 \mathrm{mM} \mathrm{CaCl}_{2} \mathrm{pH} 8$ buffer, and (c) in the presence of $500 \mathrm{mM} \mathrm{KI}$ and $0.5 \mathrm{mM} \mathrm{CaCl}_{2}$. The intensities along the lines shown in (a-c) are plotted in (d). The average intensities of the line scans from images (a) and (b) are the same, indicating that fluorophore is conserved. In the presence of KI, the intensity drops to about half of what it was initially. Also, the ratio of intensities in the $\mathrm{PA}$-rich region and $\mathrm{PC}$-rich region are the same in the presence and absence of KI. When KI was rinsed out of the bulk solution, the bilayer intensity returned to what it was prior to quenching, although more phase separation was induced (data not shown). 

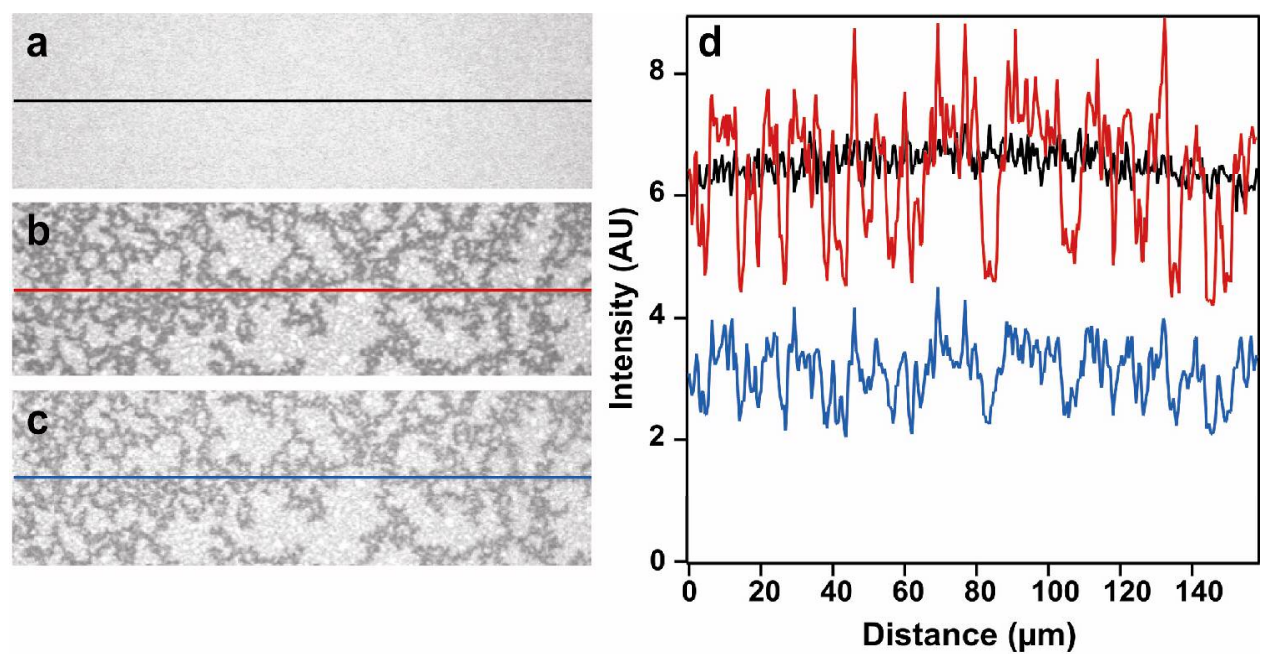

Figure S2: Bilayers composed of $30 \mathrm{~mol} \%$ DOPA, $69 \mathrm{~mol} \%$ DOPC, and $1 \mathrm{~mol} \%$ Texas Red were formed and imaged (a). The bulk solution was exchanged with a $50 \mathrm{mM} \mathrm{KCl}$, $0.5 \mathrm{mM} \mathrm{CaCl}_{2}$ buffer, $\mathrm{pH} 8$ to induce phase separation (b). The bilayer was then quenched with $500 \mathrm{mM} \mathrm{KI}, 0.5 \mathrm{mM} \mathrm{CaCl}_{2} \mathrm{pH} 8$ (c). The bilayer intensities along the lines shown in (a-c) are plotted in (d). Images (a-c) are $160 \mu \mathrm{m} \times 48 \mu \mathrm{m}$.

\section{Supplemental Tables}

Table S1: Percent Area Coverage and Percent Depletion

\begin{tabular}{cccc}
\hline$[\mathrm{KCl}](\mathrm{mM})$ & {$\left[\mathrm{CaCl}_{2}\right](\mu \mathrm{M})$} & $\%$ Area & $\%$ Depletion \\
\hline 0 & 125 & $41 \pm 5 \%$ & $22 \pm 3 \%$ \\
& 250 & $47 \pm 4 \%$ & $21 \pm 1 \%$ \\
50 & 500 & $46 \pm 5 \%$ & $15 \pm 2 \%$ \\
& 125 & $24 \pm 3 \%$ & $19 \pm 4 \%$ \\
& 250 & $30 \pm 6 \%$ & $17 \pm 4 \%$ \\
100 & 500 & $41 \pm 2 \%$ & $17 \pm 1 \%$ \\
& 125 & $10 \pm 5 \%$ & $17 \pm 1 \%$ \\
\multirow{3}{*}{250} & 250 & $28 \pm 6 \%$ & $20 \pm 4 \%$ \\
& 500 & $36 \pm 3 \%$ & $18 \pm 3 \%$ \\
& 125 & $0 \pm 0 \%$ & $0 \pm 0 \%$ \\
& 250 & $6 \pm 3 \%$ & $21 \pm 4 \%$ \\
\hline
\end{tabular}

Percent area coverage and percent depletion calculated for a variety of potassium and calcium concentrations at $\mathrm{pH}$ 8. The percent area coverage increases with decreasing potassium and increasing calcium concentrations. The percent depletion varies from 15$22 \%$, but does not seem to follow a consistent trend. 